\title{
A Leader-based Containment Control Strategy for Multiple Unicycles
}

\author{
Dimos V. Dimarogonas, Magnus Egerstedt and Kostas J. Kyriakopoulos
}

\begin{abstract}
In this paper, a leader based containment control strategy for multiple unicycle agents is introduced. Similar results for the single integrator case examined in [7] are derived based on the theory of Partial Difference Equations on graphs established in [1]. The leaders converge to a desired formation based on a control law that is independent of the followers' states. Once the leaders have reached the desired formation, the followers converge to the convex hull of the leaders final positions. When the desired leader formation is infeasible, then (as was shown in [5]) the leaders converge to a configuration where they share the same velocities and orientations. We show in this paper that in such a situation, the followers converge to the same velocities and orientations as the leaders, with the same control law that was used for the followers in the initial containment control problem. The theoretical results are verified through computer simulations.
\end{abstract}

\section{INTRODUCTION}

Cooperative control of multiple autonomous agents is a field that has gained increased attention in the past few years in both the robotics and control communities, due to the need for autonomous control of multiple mobile robotic agents sharing the same workspace. Applications include UAV formation control ([21]), micro-robotic systems([11]) and transportation systems ([24]).

Among the various specifications that the control design aims to impose on the multi-agent team, convergence of the multi-agent system to a desired formation is a design objective that has been extensively pursued during the last few years. The main feature of formation control is the cooperative nature of the equilibria of the system. Agents must converge to a desired configuration encoded by the relative inter-agent positions. Many feedback control schemes that achieve formation stabilization to a desired formation in a distributed manner have been proposed in literature. See for example [16],[14],[9] for some recent results.

The so-called agreement or rendezvous problem, in which agents must converge to the same point in the state space ([18],[4],[12], [17],[15]), is also an issue of particular interest. There have been many approaches to the state agreement problem addressing the control design issue for several vehicle models. In most cases, single integrator models of motion are taken into account, while the information exchange topology has been considered both static and dynamic, as well as bidirectional or unidirectional. A recent review of

Dimos Dimarogonas and Kostas Kyriakopoulos are with the Control Systems Lab, Department of Mechanical Engineering, National Technical University of Athens, 9 Heroon Polytechniou Street, Zografou 15780, Greece \{ddimar,kkyria@mail.ntua.gr\}. Magnus Egerstedt is with the School of Electrical and Computer Engineering, Georgia Institute of Technology, Atlanta, GA 30332, USA magnus @ece. gatech . edu the various approaches of the state agreement problem for linear models of motion is given in [20].

In this paper, a leader-based containment control problem for multiple unicycles is investigated. The problem statement can be interpreted as a combination of the formation and the agreement control problems. Specifically, the leaders of the team have two performance objectives. The first is convergence to a desired formation configuration encoded by the final desired relative inter-leader positions. The second objective is containment of the followers in the convex hull of the leaders' final positions. A similar problem was treated in [7] for multiple agents with single integrator kinematics. Unlike the strategy proposed in [7], the followers do not have to stay in the convex hull of the leaders' positions at each time instant. However, once the leaders reach the desired formation, we provide sufficient conditions for convergence of the followers to the convex hull of the leaders' final positions. This result was also established in [7] for the single integrator kinematics case. In that paper, recent results on Partial Difference Equations ([6],[1]) on graphs were used to show that, in the case of multiple stationary leaders, the followers converge to the convex hull of the leaders positions. This was achieved under an agreement control design together with some additional connectivity assumptions. Furthermore, the leaders converge to the desired formation with a control strategy that is shown to be independent of the states (positions,orientations) of the followers.

When the desired leader formation is infeasible, then (as was shown in [5]) the leaders converge to a configuration where they share the same velocities and orientations. We show in this paper that in such a situation, the followers converge to the same velocities and orientations as the leaders, with the same control law that was used for the followers in the initial containment control problem.

The nonholonomic control strategy we use in this paper for the formation control of the leaders has been used in [5], and is based on the discontinuous time-invariant control law proposed in [23]. We also use a similar control design for the followers. Time invariant controllers for nonholonomic systems have in general better convergence properties than time-varying ones. An experimental comparison between these two types of nonholonomic controllers that supports our preference to time-invariant strategies has appeared in [13]. In that reference, it was deduced that time varying controllers were too slow and oscillatory for most practical situations. On the other hand, time-invariant controllers achieved a significantly better performance.

The rest of the paper is organized as follows: Section II describes the system and states the problem treated in 
this paper. Elements from nonsmooth analysis used in the stability analysis of the proposed framework are included in Section III. Section IV presents the control design used for the followers to converge to the convex hull of the leaders, while in Section V, the formation control strategy for the leaders is described. Computer simulations that support the theoretical results are contained in Section VI, while Section VII summarizes the results of this work and indicates possible extensions.

\section{System And Problem Definition}

Consider a system of $N$ nonholonomic point agents operating in the same workspace $W \subset \mathbb{R}^{2}$. Let $q_{i}=\left[x_{i}, y_{i}\right]^{T} \in$ $\mathbb{R}^{2}$ denote the position of agent $i$. The configuration space is spanned by $q=\left[q_{1}, \ldots, q_{N}\right]^{T}$. Each agent has a specific orientation $\theta_{i}$ with respect to the global coordinate frame. The orientation vector of the agents is denoted by $\theta=$ $\left[\theta_{1} \ldots \theta_{N}\right]$. The configuration of each agent is denoted by $p_{i}=\left[\begin{array}{ll}q_{i}{ }^{T} & \theta_{i}\end{array}\right]^{T} \in \mathbb{R}^{2} \times(-\pi, \pi]$. The agent motion is described by the following nonholonomic kinematics:

$$
\begin{aligned}
& \dot{x}_{i}=u_{i} \cos \theta_{i} \\
& \dot{y}_{i}=u_{i} \sin \theta_{i} \\
& \dot{\theta}_{i}=\omega_{i},
\end{aligned}
$$

where $u_{i}, \omega_{i}$ denote the translational and rotational velocities of agent $i$, respectively. These are considered as the control inputs of the multi-agent system. We moreover assume that the agents belong to either the subset of leaders $N_{l}$, or to the subset of followers $N_{f}$, i.e. $N_{l} \bigcap N_{f}=\emptyset$ and $N_{l} \cup N_{f}=\mathcal{N}$.

The first objective of each leader is to converge to a desired formation configuration with respect to the rest of the leaders. We assume that each leader is assigned to a specific subset $N_{i}^{l} \subseteq N_{l}$ of the rest of the leaders, called leader $i$ 's leader communication set with which it can communicate in order to achieve the desired formation. The objective of each leader $i$ is to be stabilized in a desired relative position $c_{i j}$ with respect to each member $j$ of $N_{i}^{l}$. It is moreover assumed that the communication topology is bidirectional in the sense that $j \in N_{i}^{l} \Leftrightarrow i \in N_{j}^{l}, \forall i, j \in N_{l}, i \neq j$ and that the formation is feasible in the sense that $c_{i j}=-c_{j i}, \forall i, j \in N_{l}, i \neq j$. Denote $c_{i i}=-\sum_{j \in N_{i}^{l}} c_{i j}$ and $c_{l}=\left[c_{11}, \ldots, c_{N N}\right]^{T}$. In the sequel, we will use the decoupling of the stack vector $q=$ $[x, y]^{T}$ and the vector $c_{l}=\left[c_{x}, c_{y}\right]^{T}$ into the coefficients that correspond to the $x, y$ directions of the agents respectively.

Moreover, the leaders should be able to "drag" the followers along so that the latter are "contained" within the convex hull of the leader positions in the final formation configuration. This is a sub-case of the containment control problem in multi-agent systems. This problem has also been encountered in [7]. The reader is referred to that reference for a discussion on specific applications of this problem.

Both the leaders and the followers are assigned to a specific subset $N_{i} \subseteq \mathcal{N}$ of the rest of the team called agent $i$ 's leader-follower communication set with which it can communicate in order to achieve the desired objective (containment of the followers in the convex hull of the desired leader formation). We assume that for each leader $i \in N_{l}$, the sets $N_{i}, N_{i}^{l}$ are disjoint, i.e. $N_{i} \cap N_{i}^{l}=\emptyset, \forall i \in$ $N_{l}$. Hence, for the second objective, the leader follower communication set of each leader contains only followers.

These two objectives are encoded by two different communication graphs, that are defined with respect to the limited communication of the agents as follows:

1) The Leader communication graph $G^{l}=\left\{V^{l}, E^{l}, C\right\}$ is an undirected graph that consists of (i) a set of vertices $V^{l}=N_{l}$ indexed by the leaders of the multi-agent team, (ii) a set of edges, $E^{l}=\left\{(i, j) \in V^{l} \times V^{l} \mid i \in\right.$ $\left.N_{j}^{l}\right\}$ containing pairs of nodes that represent interleader formation specifications and (ii) a set of labels $C=\left\{c_{i j}\right\}$, where $(i, j) \in E^{l}$, that specify the desired inter-agent relative positions in the leader formation configuration.

2) The Leader-follower communication graph $G=$ $\{V, E\}$ is an undirected graph that consists of a set of vertices $V=\{1, \ldots, N\}$ indexed by the team members and (ii) a set of edges, $E=\left\{(i, j) \in V \times V \mid i \in N_{j}\right\}$ containing pairs of nodes that represent inter-agent communication specifications.

As an example, suppose that for a seven-agent team whose members are indexed by $1, \ldots, 7$, we have $N_{l}=$ $\{1,2,3\}, N_{f}=\{4,5,6,7\}$ and the communication sets are defined as $N_{1}^{l}=\{2\}, N_{2}^{l}=\{1,3\}, N_{3}^{l}=\{2\}$ and $N_{1}=\{4,5\}, N_{2}=\{5\}, N_{3}=\{6,7\}, N_{4}=\{1\}, N_{5}=$ $\{1,2,6\}, N_{6}=\{3,5,7\}, N_{7}=\{3,6\}$. The Leader communication graph and the Leader-follower communication graph corresponding to these communication sets are shown in Figure 1.
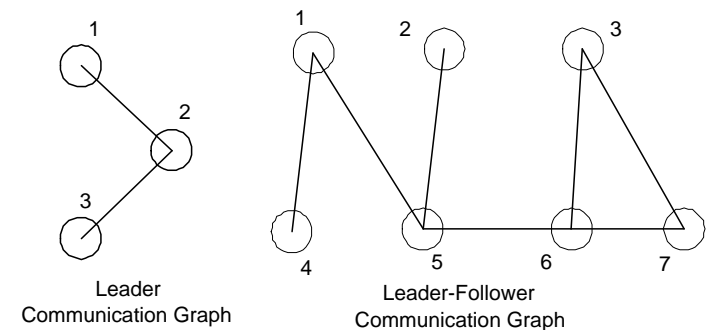

Fig. 1. Leader communication graph and Leader-follower Communication graph of a seven agent team with $N_{l}=\{1,2,3\}, N_{f}=\{4,5,6,7\}$ and communication sets $N_{1}^{l}=\{2\}, N_{2}^{l}=\{1,3\}, N_{3}^{l}=\{2\}, N_{1}=$ $\{4,5\}, N_{2}=\{5\}, N_{3}=\{6,7\}, N_{4}=\{1\}, N_{5}=\{1,2,6\}, N_{6}=$ $\{3,5,7\}, N_{7}=\{3,6\}$.

\section{TOOLS FROM Nonsmooth ANALYSIS}

In this subsection, we review some elements of nonsmooth analysis and Lyapunov theory for nonsmooth systems that we will use in the stability analysis in the next section.

For a differential equation with discontinuous right-hand side we have the following definition:

Definition 1: [8] In the case when the state-space is finite dimensional, the vector function $x($.$) is called a Filippov$ solution of $\dot{x}=f(x)$ if it is absolutely continuous and $\dot{x} \in$ 
$K[f](x)$ almost everywhere where

$$
K[f](x) \equiv \overline{c o}\left\{\lim _{x_{i} \rightarrow x} f\left(x_{i}\right) \mid x_{i} \notin \mathbf{N}\right\}
$$

where $\mathbf{N}$ is a set of measure zero.

Lyapunov stability theorems have been extended for nonsmooth systems in [22],[2]. The following chain rule provides a calculus for the time derivative of the energy function in the nonsmooth case:

Theorem 1: [22] Let $x$ be a Filippov solution to $\dot{x}=f(x)$ on an interval containing $t$ and $V: \mathbb{R}^{n} \rightarrow \mathbb{R}$ be a Lipschitz and regular function. Then $V(x(t))$ is absolutely continuous, $(d / d t) V(x(t))$ exists almost everywhere and

$$
\frac{d}{d t} V(x(t)) \in^{a . e .} \dot{\tilde{V}}(x):=\bigcap_{\xi \in \partial V(x(t))} \xi^{T} K[f](x(t))
$$

where "a.e." stands for "almost everywhere".

In this theorem, $\partial V$ is Clarke's generalized gradient. The definition of the generalized gradient and of the regularity of a function can be found in [3]. In the case we encounter in this paper, the candidate Lyapunov function $V$ we will use is smooth and hence regular, while its generalized gradient is a singleton which is equal to its usual gradient everywhere in the state space: $\partial V(x)=\{\nabla V(x)\} \forall x$.

We shall use the following nonsmooth version of LaSalle's invariance principle to prove the convergence of the prescribed system:

Theorem 2: [22] Let $\Omega$ be a compact set such that every Filippov solution to the autonomous system $\dot{x}=$ $f(x), x(0)=x\left(t_{0}\right)$ starting in $\Omega$ is unique and remains in $\Omega$ for all $t \geq t_{0}$. Let $V: \Omega \rightarrow \mathbb{R}$ be a time independent regular function such that $v \leq 0 \forall v \in \dot{\widetilde{V}}$ (if $\dot{\vec{V}}$ is the empty set then this is trivially satisfied). Define $S=\{x \in \Omega \mid 0 \in \dot{\widetilde{V}}\}$. Then every trajectory in $\Omega$ converges to the largest invariant set $M$ in the closure of $S$.

\section{Multiple Leaders}

\section{A. Multiple Stationary Leaders}

In this section, we consider the case of stationary leaders. We propose a control strategy for the followers based on their information imposed by the leader-follower communication graph that guarantees convergence of the followers to the convex hull of the leaders.

The control design for each follower will be of the form

$$
\begin{aligned}
& u_{i}=u_{i}\left(p_{i}, p_{j}\right) \\
& \omega_{i}=\omega_{i}\left(p_{i}, p_{j}\right)
\end{aligned}, j \in N_{i}, i \in N_{f}
$$

copying in this way with the limited communication capabilities of each follower. In the sequel, the notation $(a)_{i}$ for a vector $a$, denotes its $i$-th element. Let also $L$ denote the Laplacian matrix of the leader-follower communication graph ([10]). The following theorem guarantees convergence of the followers to the convex hull of the stationary leaders:

Theorem 3: Assume that the Leader-follower communication graph is connected and that the subset of leaders is nonempty. Then the discontinuous time-invariant feedback control strategy:

$$
u_{i}=-\operatorname{sgn}\left(\gamma_{x i} \cos \theta_{i}+\gamma_{y i} \sin \theta_{i}\right) \cdot\left(\gamma_{x i}^{2}+\gamma_{y i}^{2}\right)^{1 / 2}
$$

$$
\omega_{i}=-\left(\theta_{i}-\theta_{n h_{i}}\right)
$$

where $\gamma_{x i}=(L x)_{i}, \gamma_{y i}=(L y)_{i}$, and the "nonholonomic angle" $\theta_{n h_{i}}=\arctan 2\left(\gamma_{y i}, \gamma_{x i}\right)$, drives the followers to the convex hull of the leaders positions.

Proof: Define $\gamma_{i}=\frac{1}{2} \sum_{j \in N_{i}}\left\|q_{i}-q_{j}\right\|^{2}, i \in N$. Then it is easily seen that $\sum_{i \in N} \nabla \gamma_{i}=2\left(L \otimes I_{2}\right) q$, where $\otimes$ denotes the standard Kronecker product between two matrices.

We shall use the smooth positive definite function $V=$ $\sum_{i \in N} \gamma_{i}$ as a candidate Lyapunov function. Since the proposed control law is discontinuous we use Theorem 1 for the time derivative of the candidate Lyapunov function. Since $V$ is smooth we have $\partial V=\{\nabla V\}=\left\{\sum_{i \in N} \nabla \gamma_{i}\right\}$, so that

$$
\begin{aligned}
& \left.V=\sum_{i \in N} \gamma_{i} \Rightarrow \dot{\widetilde{V}}=\left\{\sum_{i \in N}\left(\nabla \gamma_{i}\right)^{T}\right\}\right\} K\left[\begin{array}{c}
u_{1} \cos \theta_{1} \\
u_{1} \sin \theta_{1} \\
\vdots \\
u_{N} \cos \theta_{N} \\
u_{N} \sin \theta_{N}
\end{array}\right] \subset \\
& 2 q^{T}\left(L \otimes I_{2}\right)\left[\begin{array}{c}
K\left[u_{1}\right] \cos \theta_{1} \\
K\left[u_{1}\right] \sin \theta_{1} \\
\vdots \\
K\left[u_{N}\right] \cos \theta_{N} \\
K\left[u_{N}\right] \sin \theta_{N}
\end{array}\right] \subset \\
& 2(L x)^{T}\left[\begin{array}{c}
K\left[u_{1}\right] \cos \theta_{1} \\
\vdots \\
K\left[u_{N}\right] \cos \theta_{N}
\end{array}\right]+2(L y)^{T}\left[\begin{array}{c}
K\left[u_{1}\right] \sin \theta_{1} \\
\vdots \\
K\left[u_{N}\right] \sin \theta_{N}
\end{array}\right] \\
& \subset \sum_{i \in N_{f}}\left\{2 K\left[u_{i}\right]\left((L x)_{i} \cos \theta_{i}+(L y)_{i} \sin \theta_{i}\right)\right\}
\end{aligned}
$$

where the summation is held over the set of followers since the leaders are assumed stationary. We also used Theorem 1.3 in [19] to calculate the inclusions of the Filippov set in the previous analysis. Since $K[\operatorname{sgn}(x)] x=\{|x|\}([19]$, Theorem $1.7)$, the choice of control laws (3),(4) results in

$\dot{\widetilde{V}}=-2 \sum_{i \in N_{f}}\left\{\left(\gamma_{x i}^{2}+\gamma_{y i}^{2}\right)^{1 / 2} \cdot\left|\gamma_{x i} \cos \theta_{i}+\gamma_{y i} \sin \theta_{i}\right|\right\} \leq 0$

so that the generalized derivative of $V$ reduces to a singleton.

Since the candidate Lyapunov function is quadratic in the agents' relative positions, its level sets are compact and invariant for the trajectories of the closed loop system. Specifically, we have $V \leq c \Rightarrow\left\|q_{i}-q_{j}\right\| \leq \sqrt{2 c}, \forall(i, j) \in E$. Connectivity of the Leader-follower communication graph ensures that the maximum length of a path connecting two vertices of the graph is at most $N-1$. Hence $\left\|q_{i}-q_{j}\right\| \leq$ $\sqrt{2 c}(N-1), \forall i, j \in \mathcal{N}$.

Now, using the nonsmooth version of LaSalle's invariance principle (Theorem 2), we conclude that the followers converge to the largest invariant subset of the set

$$
S=\left\{\begin{array}{r}
\left(\gamma_{x i}=\gamma_{y i}=0\right) \vee\left(\gamma_{x i} \cos \theta_{i}+\gamma_{y i} \sin \theta_{i}=0\right), \\
\forall i \in N_{f}
\end{array}\right\}
$$

However, for each $i \in N_{f}$, we have $\left|\omega_{i}\right|=\frac{\pi}{2}$ whenever $\gamma_{x i} \cos \theta_{i}+\gamma_{y i} \sin \theta_{i}=0$, due to the definition of $\theta_{n h_{i}}$. 
Hence the largest invariant set contained in $S$ is

$$
S \supset S_{0}=\left\{\gamma_{x i}=\gamma_{y i}=0, \forall i \in N_{f}\right\}
$$

In this set, the orientations of all followers converge to zero, since $\theta_{n h_{i}}=0$ for each follower $i$ in $S_{0}$. Hence, the system converges to a configuration that is an equilibrium of the partial difference equation

$$
\begin{aligned}
& (L x)_{i}=(L y)_{i}=0, \forall i \in N_{f} \\
& q^{l}=q^{l}(0)
\end{aligned}
$$

where $q^{l}$ is the stack vector of the leaders' configuration. The solutions of (5) have been studied in [7]. In particular, Theorem 2 in [7] states that for a connected Leader-follower communication graph and a nonempty set of leaders, the position of each follower, as given by the solution of (5), lies in the convex hull of the leaders' positions. Hence the proof is complete. $\diamond$

\section{B. Multiple Moving Leaders}

Theorem 3 assumes that the leaders are stationary, namely that $\dot{q}_{i}=0, \forall i \in N_{l}$. In this section, we examine the case when the leaders move with the same velocities and orientations. Hence the leaders' motion is described by

$$
\dot{x}_{i}=u_{x}, \dot{y}_{i}=u_{y}, \dot{\theta}_{i}=0, \forall i \in N_{l}
$$

The following theorem guarantees that the followers converge to a configuration where they have the same velocities and orientations as the leaders:

Theorem 4: Assume that the Leader-follower communication graph is connected and that the subset of leaders is nonempty. Assume also that the followers evolve under the control law (3),(4) while the leaders move according to (6). Then the followers converge to a configuration where they have the same velocities and orientations as the leaders.

Proof: Equation (4) implies that $\theta_{i}$ is aligned with $\theta_{n h_{i}}$ as $t \rightarrow \infty$. Hence, in steady state, the followers'motion in the $x, y$-coefficients under the control laws (3),(4) is given by

$$
\dot{x}_{i}=u_{i} \cos \theta_{n h_{i}}=-\operatorname{sgn}\left\{\gamma_{x i} \cos \theta_{n h_{i}}+\gamma_{y i} \sin \theta_{n h_{i}}\right\} \gamma_{x i}
$$$$
\dot{y}_{i}=u_{i} \sin \theta_{n h_{i}}=-\operatorname{sgn}\left\{\gamma_{x i} \cos \theta_{n h_{i}}+\gamma_{y i} \sin \theta_{n h_{i}}\right\} \gamma_{y i}
$$

But, by definition of $\theta_{n h_{i}}$, we have $\gamma_{x i} \cos \theta_{n h_{i}}+$ $\gamma_{y i} \sin \theta_{n h_{i}}>0$. Then, at steady state, we have:

$$
\begin{aligned}
& \dot{x}_{i}=-\gamma_{x i} \\
& \dot{y}_{i}=-\gamma_{y i}
\end{aligned}, i \in N_{f}
$$

Using $W=\frac{1}{2}\left(\dot{x}^{T} L \dot{x}+\dot{y}^{T} L \dot{y}\right)$ as a candidate Lyapunov function for the system (7) and differentiating with respect to time we get:

$$
\dot{W}=\dot{x}^{T} L \ddot{x}+\dot{y}^{T} L \ddot{y}=-\sum_{i \in N_{f}}\left(\left(\dot{x}^{T} L\right)_{i} \dot{\gamma}_{x i}+\left(\dot{y}^{T} L\right)_{i} \dot{\gamma}_{y i}\right)
$$

since $\ddot{x}_{i}=\ddot{y}_{i}=0, \forall i \in N_{l}$. We also have $\dot{\gamma}_{x i}=$ $(L \dot{x})_{i}, \dot{\gamma}_{y i}=(L \dot{y})_{i}$ so that

$$
\dot{W}=-\sum_{i \in N_{f}}\left((L \dot{x})_{i}^{2}+(L \dot{y})_{i}^{2}\right) \leq 0
$$

Following similar arguments as in the proof of the previous Theorem, it is easily shown that the level sets of $W$ are compact and invariant with respect to the agents' relative velocity components. Using Lasalle's invariance principle, we deduce that the agents converge to a configuration that is an equilibrium of the partial difference equation

$$
\begin{aligned}
& (L \dot{x})_{i}=(L \dot{y})_{i}=0, \forall i \in N_{f} \\
& \dot{x}_{i}=u_{x}, \dot{y}_{i}=u_{y}, \forall i \in N_{l}
\end{aligned}
$$

Similarly to the proof of Theorem 3 , we deduce that the velocity of each follower, as given by the solution of (8), lies in the convex hull of the leaders' velocities. Since the convex hull of the leaders velocities reduces to the singleton $\left\{u_{x}\right\}$ in the $x$-direction and to the singleton $\left\{u_{y}\right\}$ in the $y$-direction, it is straightforward that at steady state,

$$
\dot{x}_{i}=u_{x}, \dot{y}_{i}=u_{y}, \forall i \in N_{f}
$$

Furthermore, since for all $i \in N_{f}$, we have

$$
\begin{aligned}
& \dot{x}_{i}=u_{x}=-\gamma_{x i}, \dot{y}_{i}=u_{y}=-\gamma_{y i}, \\
& \theta_{i}=\theta_{n h_{i}}=\arctan 2\left(-\gamma_{y i},-\gamma_{x i}\right)
\end{aligned}
$$

and the common orientation of the leaders is also given by

$$
\arctan 2\left(u_{y i}, u_{x i}\right)=\arctan 2\left(-\gamma_{y i},-\gamma_{x i}\right)
$$

we deduce that all agent converge to a common orientation. Hence the proof is complete. $\diamond$

\section{LEADER FORMATION CONTROL DESIGN}

\section{A. Convergence to a feasible formation}

From the proof of the previous section, it is straightforward to see that the leader formation control strategy can be designed in a totally independent manner with respect to the followers. Once the leaders converge to the desired formation, Theorem 3 guarantees that the followers move to the convex hull of the leader formation, under the control strategy (3),(4). In essence, the control law of each leader is able to contain information based solely on the Leader communication graph.

Let $L^{l}$ denote the Laplacian matrix of the Leader formation graph and $x^{l}, y^{l}$ denote the decoupling of the stack vector of the leaders $q^{l}=\left[x^{l}, y^{l}\right]^{T}$ into the coefficients that correspond to the $x, y$ directions of the leaders respectively. The following theorem guarantees convergence of the leaders to the desired formation:

Theorem 5: Assume that the Leader communication graph is connected. Then the control design

$$
\begin{gathered}
u_{i}=-\operatorname{sgn}\left(\gamma_{x i}^{l} \cos \theta_{i}+\gamma_{y i}^{l} \sin \theta_{i}\right) \cdot\left(\left(\gamma_{x i}^{l}\right)^{2}+\left(\gamma_{y i}^{l}\right)^{2}\right)^{1 / 2} \\
\omega_{i}=-\left(\theta_{i}-\theta_{n h_{i}}^{l}\right)
\end{gathered}
$$

where $\gamma_{x i}^{l}=\left(L^{l} x^{l}+c_{x}\right)_{i}, \gamma_{y i}^{l}=\left(L^{l} y^{l}+c_{y}\right)_{i}$ and $\theta_{n h_{i}}^{l}=$ $\arctan 2\left(\gamma_{y i}^{l}, \gamma_{x i}^{l}\right)$, drives the leaders to the desired leader formation.

Proof: Define the term $\gamma_{i}^{l}=\frac{1}{2} \sum_{j \in N_{i}^{l}}\left\|q_{i}-q_{j}-c_{i j}\right\|^{2}, i \in$ $N_{l}$. Similarly to the proof of the previous theorem, we have 
$\sum_{i \in N_{l}} \nabla \gamma_{i}^{l}=2\left(\left(L^{l} \otimes I_{2}\right) q^{l}+c_{l}\right)$. Using $V=\sum_{i \in N_{l}} \gamma_{i}^{l}$ as a candidate Lyapunov function and computing its generalized time derivative we get

$$
\dot{\widetilde{V}}=\sum_{i \in N_{l}}\left\{2 K\left[u_{i}\right]\left(\begin{array}{c}
\left(L^{l} x+c_{x}\right)_{i} \cos \theta_{i}+ \\
+\left(L^{l} y+c_{y}\right)_{i} \sin \theta_{i}
\end{array}\right)\right\}
$$

With the choice of control laws (9),(10) we have

$$
\dot{\tilde{V}}=-2 \sum_{i \in N_{l}}\left\{\begin{array}{l}
\left(\left(\gamma_{x i}^{l}\right)^{2}+\left(\gamma_{y i}^{l}\right)^{2}\right)^{1 / 2} \cdot \\
\cdot\left|\left(\gamma_{x i}^{l}\right) \cos \theta_{i}+\left(\gamma_{y i}^{l}\right) \sin \theta_{i}\right|
\end{array}\right\} \leq 0
$$

Quoting again the nonsmooth version of LaSalle's invariance principle (Theorem 2), and using the same arguments as in the proof of Theorem 3, we conclude that the leaders converge to the set

$$
S_{0}^{l}=\left\{\gamma_{x i}^{l}=\gamma_{y i}^{l}=0, \forall i \in N_{l}\right\}
$$

This guarantees that the leaders converge to the desired formation configuration. This is easily derived from the fact that $\left(\gamma_{x i}^{l}=\gamma_{y i}^{l}=0\right) \forall i \Rightarrow\left(L^{l} \otimes I_{2}\right) q+c_{l}=0$. For all $i \in N_{l}$, let $c_{i}$ denote the configuration of leader $i$ in a desired leader formation configuration with respect to the global coordinate frame. It is then obvious that $c_{i j}=c_{i}-c_{j}$, for all $(i, j) \in E^{l}$, for all possible desired final formations. Define $q_{i}-q_{j}-c_{i j}=q_{i}-q_{j}-\left(c_{i}-c_{j}\right)=\tilde{q}_{i}-\tilde{q}_{j}$. Then we have that $\left(L^{l} \otimes I_{2}\right) q+c_{l}=0 \Rightarrow\left(L^{l} \otimes I_{2}\right) \tilde{q}=0 \Rightarrow L^{l} \tilde{x}=L^{l} \tilde{y}=0$ where $\tilde{x}, \tilde{y}$ the stack vectors of $\tilde{q}$ in the $x, y$ directions. The fact that the leader communication graph is connected implies that both $\tilde{x}, \tilde{y}$ are eigenvectors of $L^{l}$ belonging to $\operatorname{span}\{\overrightarrow{\mathbf{1}}\}$. Therefore all $\tilde{q}_{i}$ are equal to a common vector value $c$. Hence $\tilde{q}_{i}=c \forall i \Rightarrow q_{i}-q_{j}=c_{i j} \forall i, j \in N_{l}, j \in N_{i}^{l}$.

We conclude that the leaders converge to the desired relative configuration. $\diamond$

\section{B. The case of formation infeasibility}

Theorem 5 involves convergence of the leaders to a desired formation, and is applied along with the result of Theorem 3 to the problem of containing the group of followers within the convex hull of the final leader formation. Theorem 4 on the other hand, assumes that the leaders have a common, possibly non-zero, velocity and a common orientation. The next result provides the means of obtaining such a behavior on the leaders' part. Specifically, in our previous work [5] it was shown that formation infeasibility forces the leaders to converge to a configuration where they share a common velocity and orientation. Formation infeasibility is implied by the assumption that the condition $c_{i j}=-c_{i j}, \forall i, j \in$ $N_{l}, i \neq j$ is not guaranteed to hold for the desired leaders' relative positions. The desired leader formation might be rendered infeasible in this way, in the sense that the set $\left\{q: q_{i}-q_{j}=c_{i j}, \forall(i, j) \in E^{l}\right\}$ may be empty.

The following result was presented in [5]:

Theorem 6: Assume that the Leader communication graph is connected. Then the control design (9),(10) drives the system to a configuration in which all leaders have the same velocities and orientations even if the desired leader formation configuration is infeasible.
Proof: The proof of this statement can be found in [5]. It follows the same arguments of the proof of Theorem $4 . \diamond$

\section{Simulations}

To verify the results of the previous paragraphs, we provide two computer simulations of the proposed framework.

The first simulation involves a multi-agent team of six agents, three leaders (red) and three followers (blue). The leaders aim to converge to a triangular formation, while the followers inside the convex hull. The leaders evolve under the control laws (9),(10) while the followers under the control law (3),(4). Screenshots I-IV in Figure 2 show the evolution in time of the unicycle group. The leaders are denoted by "L", while the followers by "F". The red leaders converge to the desired formation, while the followers in the convex hull of the leaders final positions (i.e. inside the triangle). This can be witnessed in the last screenshot IV. The communication sets of both leaders and followers are chosen so that they fulfill the desired connectivity properties imposed by theorems 3,5 .
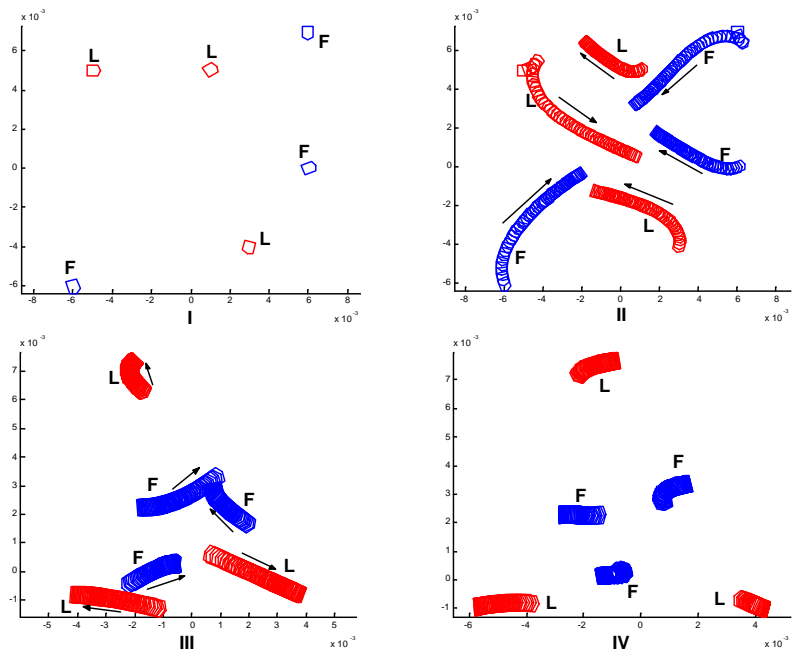

Fig. 2. Simulation A

In the next simulation, we have again three leaders(red), denoted by "L" and three followers(blue), denoted by "F". The communication sets of both leaders and followers are chosen so that they fulfill the desired connectivity properties imposed by theorems 4, 6. The desired leader formation is now rendered infeasible with an appropriate choice of the inter-leader relative positions. Screenshots I-V in figure 3 show the evolution in time of the unicycle group. In the last screenshot $\mathrm{V}$, all agents' velocities and orientations converge to a common value, something also witnessed in the velocity plot figure 4 .

\section{CONCLUSIONS}

In this paper, a leader based containment control strategy for multiple unicycle agents has been presented. Similar results for the single integrator case examined in [7] were derived based on the theory of Partial Difference Equations on graphs established in [1]. The leaders converge to a 


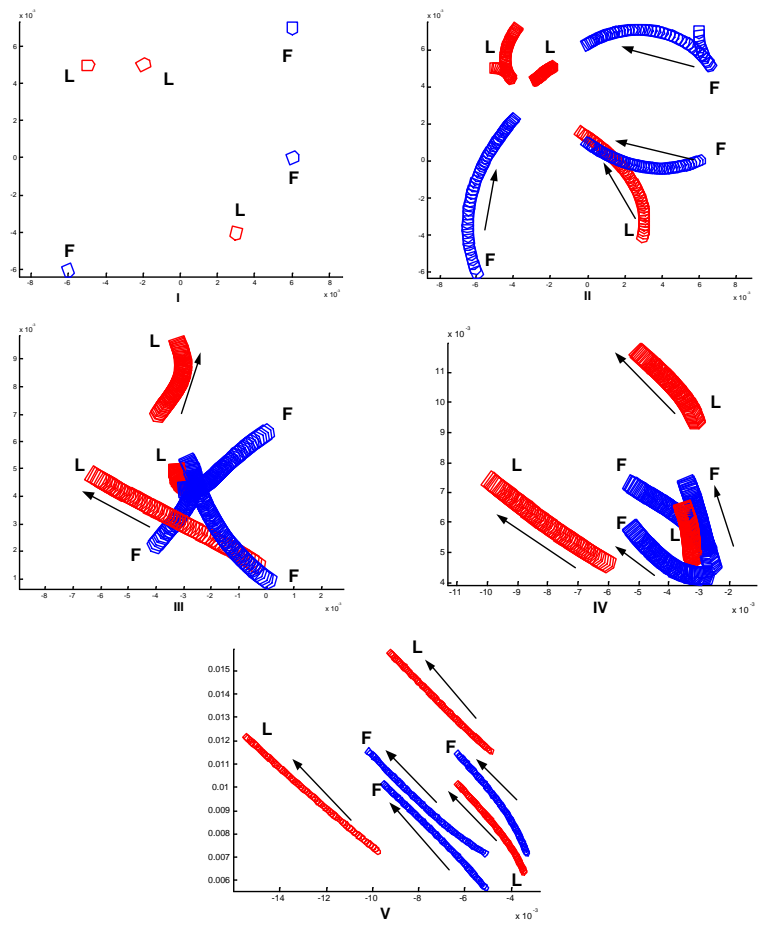

Fig. 3. Simulation B

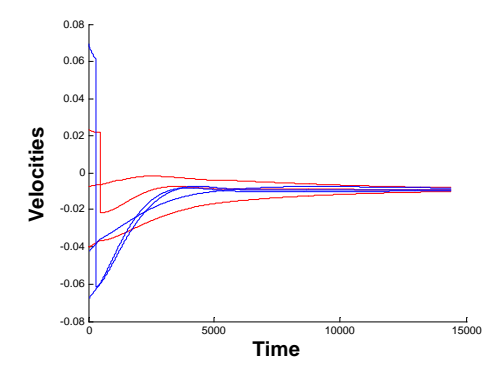

Fig. 4. Agents' velocities in Simulation B converge to a common value.

desired formation based on a control law that is independent of the followers' states. Once the leaders reach the desired formation, we showed that the followers converge to the convex hull of the leaders final positions. When the desired leader formation is infeasible, then (as was shown in [5]) the leaders converge to a configuration where they share the same velocities and orientations. We showed that in such a situation, the followers converge to the same velocities and orientations as the leaders, with the same control law that was used for the followers in the initial containment control problem. The theoretical results were verified through computer simulations.

Current research aims to take into account the case of unidirectional communication links (i.e. directed graphs), as well as taking into account collision avoidance issues between the team members.

\section{ACKNOWLEDGEMENTS}

Dimos Dimarogonas and Kostas Kyriakopoulos would like to acknowledge the contribution of the European Commis- sion through contract I-SWARM (IST-2004-507006). Magnus Egerstedt's work is sponsored by the US Army Research Office through grant No.99838.

\section{REFERENCES}

[1] A. Bensounssan and J.L. Menaldi. Difference equations on weighted graphs. Journal of Convex Analysis, 12:13-44, 2005.

[2] F. Ceragioli. Discontinuous Ordinary Differential Equations and Stabilization. PhD thesis, Dept. of Mathematics, Universita di Firenze, 1999.

[3] F. Clarke. Optimization and Nonsmooth Analysis. Addison - Wesley, 1983.

[4] J. Cortes, S. Martinez, and F. Bullo. Robust rendezvous for mobile autonomous agents via proximity graphs in arbitrary dimensions. IEEE Transactions on Automatic Control, 51(8):1289-1298, 2006.

[5] D.V. Dimarogonas and K.J. Kyriakopoulos. A connection between formation control and flocking behavior in nonholonomic multi-agent systems. IEEE Intern. Conf. Robotics and Automation, pages 940-945, 2006.

[6] G. Ferrari-Trecate, A. Buffa, and M. Gati. Analysis of coordination in multi-agent systems through partial difference equations. part i: The laplacian control. 16th IFAC World Congress, 2005.

[7] G. Ferrari-Trecate, M. Egerstedt, A. Buffa, and M. Ji. Laplacian sheep: A hybrid, stop-go policy for leader-based containment control. In Hybrid Systems: Computation and Control, pages 212-226. Springer Verlag, 2006.

[8] A. Filippov. Differential equations with discontinuous right-hand sides. Kluwer Academic Publishers, 1988.

[9] V. Gazi and K.M. Passino. Stability analysis of swarms. IEEE Transactions on Automatic Control, 48(4):692-696, 2003.

[10] C. Godsil and G. Royle. Algebraic Graph Theory. Springer Graduate Texts in Mathematics \# 207, 2001.

[11] Project ISWARM. http://microrobotics.ira.uka.de/.

[12] M. Ji and M. Egerstedt. Connectedness preserving distibuted coordination control over dynamic graphs. 2005 American Control Conference, pages 93-98.

[13] B. Kim and P. Tsiotras. Controllers for unicycle-type wheeled robots: Theoretical results and experimental validation. IEEE Transactions on Robotics and Automation, 18(3):294-307, 2002.

[14] G. Lafferriere, A. Williams, J. Caughman, and J.J.P. Veerman. Decentralized control of vehicle formations. Systems and Control Letters, 54(9):899-910, 2005.

[15] J. Lin, A.S. Morse, and B. D. O. Anderson. The multi-agent rendezvous problem. 42st IEEE Conf. Decision and Control, pages 1508-1513, 2003.

[16] Z. Lin, B. Francis, and M. Maggiore. Necessary and sufficient graphical conditions for formation control of unicycles. IEEE Transactions on Automatic Control, 50(1):121-127, 2005.

[17] L. Moreau. Stability of continuous-time distributed consensus algorithms. 43rd IEEE Conf. Decision and Control, pages 3998-4003, 2004.

[18] R. Olfati-Saber and R.M. Murray. Consensus problems in networks of agents with switching topology and time-delays. IEEE Transactions on Automatic Control, 49(9):1520-1533, 2004.

[19] B. Paden and S. S. Sastry. A calculus for computing filippov's differential inclusion with application to the variable structure control of robot manipulators. IEEE Trans. on Circuits and Systems, 34(1):7382, 1987.

[20] W. Ren, R. W. Beard, and E. M. Atkins. A survey of consensus problems in multi-agent coordination. 2005 American Control Conference, pages $1859-1864$.

[21] A. Ryan, M. Zennaro, A. Howell, R. Sengupta, and K. J. Hendrick. An overview of emerging results in cooperative uav control. 43rd IEEE Conf. Decision and Control, pages 602-607, 2004.

[22] D. Shevitz and B. Paden. Lyapunov stability theory of nonsmooth systems. IEEE Trans. on Automatic Control, 49(9):1910-1914, 1994.

[23] H. Tanner and K.J. Kyriakopoulos. Backstepping for nonsmooth systems. Automatica, 39:1259-1265, 2003.

[24] C. Tomlin, G.J. Pappas, and S. Sastry. Conflict resolution for air traffic management: A study in multiagent hybrid systems. IEEE Transactions on Automatic Control, 43(4):509-521, 1998. 